\title{
Depression and Comorbid Illness in Elderly Primary Care Patients: Impact on Multiple Domains of Health Status and Well-being
}

\author{
Polly Hitcbcock Noël, $P b D^{1,2}$ \\ Jobn W. Williams Jr, MD, MHS $S^{3,4}$ \\ Jürgen Unützer, $M D, M P H^{5}$ \\ Jason Worchel, $M D^{6}$ \\ Shuko Lee, MS \\ Jobn Cornell, $\mathrm{PbD}{ }^{1.2}$ \\ Wayne Katon, $M D^{7,8}$ \\ Linda H. Harpole, MD, MPH \\ Enid Hunkeler, $M A^{9}$ \\ 'VERDICT, an HSR\&D Center of \\ Excellence, South Texas Veterans Health \\ Care System, San Antonio, Tex \\ ${ }^{2}$ University of Texas Health Science Center, \\ San Antonio, Tex \\ ${ }^{3}$ Center for Health Services Research in \\ Primary Care, Durham Veterans Affairs \\ Medical Center, Durham, NC \\ ${ }^{4}$ Duke University Medical Center, \\ Durham, NC \\ ${ }^{5}$ University of California Los Angeles, \\ Los Angeles, Calif \\ ${ }^{6}$ Central Texas Veterans Health Care System, \\ Austin, Tex \\ ${ }^{7}$ University of Washington School of \\ Medicine, Seattle, Wash \\ ${ }^{8}$ Group Health Cooperative of Puget Sound, \\ Seattle, Wash \\ ${ }^{9}$ Kaiser Permanente of Northern California, \\ Oakland, Calif
}

Conflicts of interest: none reported

\section{CORRESPONDING AUTHOR}

Polly Hitchcock Noël, PhD

VERDICT, an HSR\&D Center of Excellence South Texas Veterans Health Care System 7400 Merton Minter Blvd (11C6)

San Antonio, TX 78229-4404

pollyh@verdict.uthscsa.edu.

\begin{abstract}
PURPOSE Our objective was to examine the relative association of depression severity and chronicity, other comorbid psychiatric conditions, and coexisting medical illnesses with multiple domains of health status among primary care patients with clinical depression.

METHODS We collected cross-sectional data as part of a treatment effectiveness trial that was conducted in 8 diverse health care organizations. Patients aged 60 years and older $(N=1,801)$ who met diagnostic criteria for major depression or dysthymia participated in a baseline survey. A survey instrument included questions on sociodemographic characteristics, depression severity and chronicity, neuroticism, and the presence of 11 common chronic medical illnesses, as well as questions screening for panic disorder and posttraumatic stress disorder. Measures of 4 general health indicators (physical and mental component scales of the SF-12, Sheehan Disability Index, and global quality of life) were included. We conducted separate mixed-effect regression linear models predicting each of the 4 general health indicators.
\end{abstract}

RESULTS Depression severity was significantly associated with all 4 indicators of general health after controlling for sociodemographic differences, other psychological dysfunction, and the presence of 11 chronic medical conditions. Although study participants had an average of 3.8 chronic medical illnesses, depression severity made larger independent contributions to 3 of the 4 general health indicators (mental functional status, disability, and quality of life) than the medical comorbidities.

CONCLUSIONS Recognition and treatment of depression has the potential to improve functioning and quality of life in spite of the presence of other medical comorbidities.

Ann Fam Med 2004;2:555-562. DOI: 10.1370/afm.143.

\section{INTRODUCTION}

E pidemiological and clinical studies consistently indicate that depression adversely affects the lives of older adults. The relative contribution to adverse effects is not entirely clear, because depression often occurs in conjunction with other psychiatric illnesses, such as anxiety disorders; somatic symptoms, such as pain ${ }_{i}$ and chronic medical illnesses, such as diabetes. The latter is particularly of concern, because it is often difficult to know whether a particular symptom, such as lethargy, is caused by depression, a coexisting medical illness, or both. Patients with chronic medical illness are known to have a high prevalence of comorbid depression. ${ }^{1}$ Furthermore, both major depressive disorder and subsyndromal depression have been associated with increased somatic symptoms, morbidity, mortality, health care utilization, and costs in the presence of comorbidities. ${ }^{1-4}$ 
Some studies have found that patients with depression have more functional impairment and poorer quality of life than patients with other chronic illnesses. ${ }^{5-7}$ Furthermore, severity of depressive symptoms is inversely related to patients' health-related quality of life, even after controlling for age, sex, and medical comorbidities. ${ }^{8,9}$ Many older persons, however, have more than one chronic illness that may differentially impair health status. Elders with multiple comorbidities may be particularly vulnerable to the debilitating impact of depression. Much of the previous research examining the interconnections between depression, medical comorbidities, and health status has been conducted in restricted settings. It is therefore difficult to compare the impact of depression with that of other chronic medical disorders to inform policy decisions about health care resource allocation. ${ }^{8}$

Although researchers have increasingly recognized the importance of including patient-centered measures of health status in outcomes research, a wide variety of concepts and measures have been used, including quality of life, functional status, and disability. Because depression and other illnesses may affect multiple dimensions of health status, simultaneous examination of these may provide a richer understanding. Using baseline data from an intervention study of 1,801 depressed elders, ${ }^{16}$ we examined the association of depression severity and chronicity, other comorbid psychiatric conditions, and coexisting medical illnesses with multiple domains of general health status. Our goal was to answer the following question: among older adults with clinical depression, what is the relative association of depression severity and chronicity with functional status, quality of life, and disability compared with comorbid psychiatric illnesses and coexisting medical illnesses?

\section{METHODS}

Project IMPACT is a multicenter randomized controlled trial comparing usual care with the effectiveness of collaborative disease management for late-life depression in primary care. ${ }^{16}$ Study protocols were approved by the institutional review boards at all sites. All patients signed an informed consent approved by their local institutional review board. Cross-sectional data collected at baseline were used for the analyses described in this report.

\section{Sample}

Eighteen participating primary care clinics belonged to a total of 8 different health care organizations in 5 states. Represented were 2 staff model health maintenance organizations (HMO), 2 regions of a large group model HMO, the Department of Veterans Affairs, 2 university-affiliated primary care clinics, and 1 private practice physician group. Recruitment procedures were developed to enroll a sample of depressed older primary care patients that could be identified for a quality improvement intervention under real-world conditions. ${ }^{16}$ Each site used 2 methods to identify study participants. The first method consisted of referrals from primary care providers, other staff, or patients themselves in response to clinic promotions of the study. The second method consisted of systematic screening using a 2 -item instrument that screened for depression. ${ }^{17}$

Inclusion criteria were age 60 years or older, intent to use one of the study clinics as the main source of primary care in the coming year, and a diagnosis of current major depression or dysthymia according to the Structured Clinical Interview for DSM-IV Axis I Disorders (SCID). ${ }^{18,19}$ Exclusions included a current drinking problem (a score of 2 or more on the CAGE questionnaire), ${ }^{20}$ a history of bipolar disorder or psychosis, ongoing psychiatric treatment, severe cognitive impairment, and acute risk of suicide. A total of 907 of the patients identified by screening and 894 of the referrals enrolled in the study (Figure 1). ${ }^{21}$

\section{Measures}

Trained lay interviewers conducted in-person structured computerized interviews before randomization. The interviews assessed sociodemographic characteristics, severity of depression symptoms using the Hopkins Symptom Checklist (HSCL-20), ${ }^{22}$ chronic depression (depressed or anhedonic more than one half of the days during past 2 years), presence of panic attacks in past 4 weeks, ${ }^{23}$ neuroticism with a 7 -item subscale of the NEO Personality Inventory, ${ }^{24}$ posttraumatic stress disorder using a 3 -item screening tool, ${ }^{25}$ presence of mild cognitive impairment using a 6-item screening tool derived from the Mini-Mental Status Examination, ${ }^{26}$ and a history of diagnosis or treatment for common chronic medical problems during the preceding 3 years. Related conditions were collapsed into 11 general categories. Patients were specifically asked about the following: asthma, emphysema, or chronic bronchitis (chronic lung disease), high blood pressure or hypertension (hypertension); high blood glucose or diabetes (diabetes); arthritis or rheumatism (arthritis), loss of hearing or vision (sensory deficit); cancer excluding skin cancer (cancer); neurological conditions, such as epilepsy, seizures, Parkinson's disease, or stroke (neurological disease), heart disease, such as angina, heart failure, or valve problems (heart disease); chronic back problems, headache, or other chronic pain problems (chronic pain); stomach ulcer, chronic inflamed bowel, enteritis, or colitis (gastrointestinal disease); and chronic problems with urination, chronic bladder infec- 


\section{Figure 1. IMPACT participant flow.}

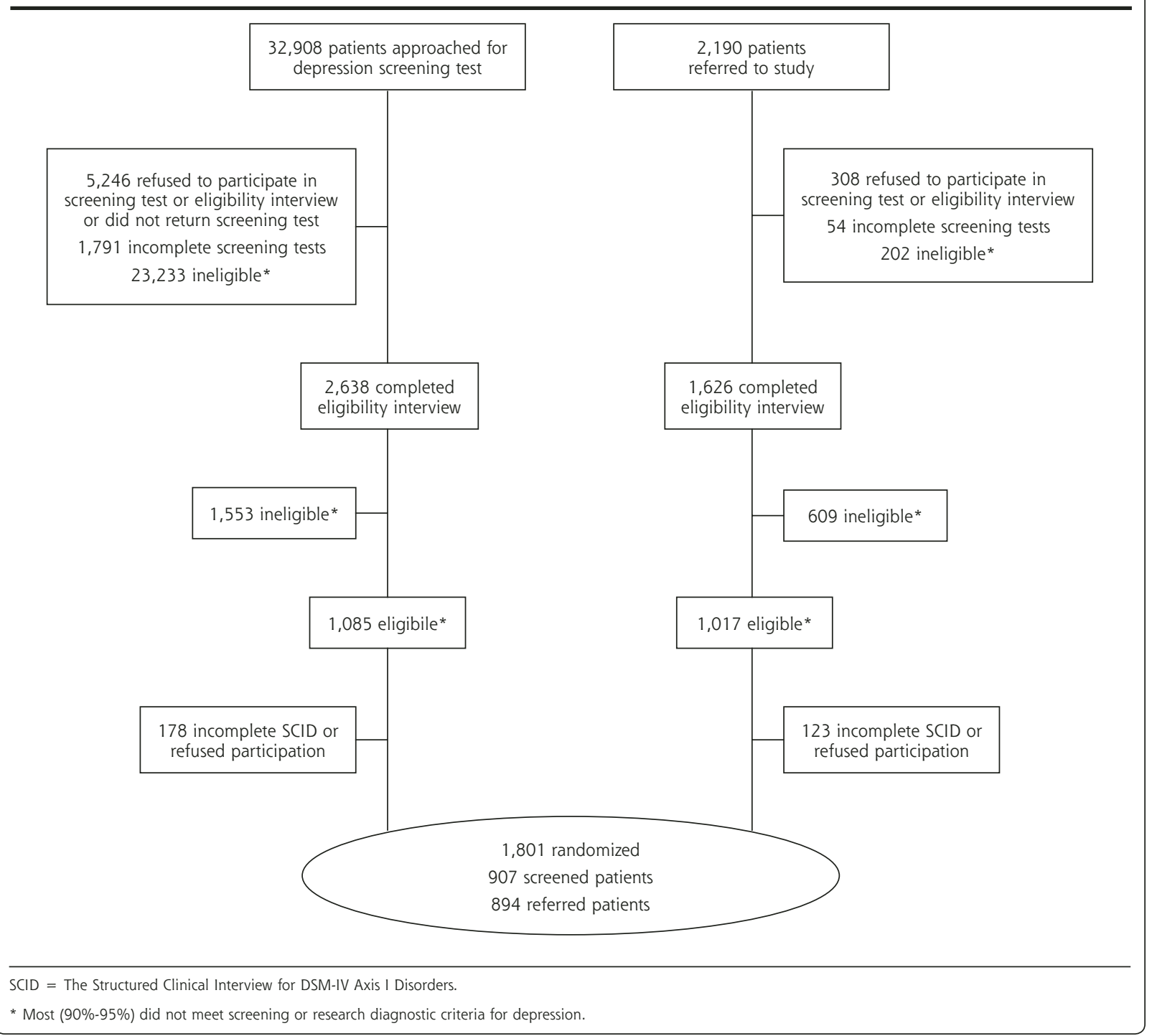

tions or prostate problems, or incontinence or inability to hold urine (urinary tract or prostate disease).

Outcome measures included the physical component score (PCS-12) and mental component score (MCS-12) of the Rand 12-item Short Form (SF-12); the normed-based scores have a mean of 50 (SD = 10) with lower scores indicating poorer functioning. ${ }^{27}$ Quality-of-life score (QOL) was measured by a singleitem rating of overall quality of life in the past month on a scale from 0 (about as bad as dying) to 10 (life is perfect).$^{28}$ Disability was measured by an index (SDI) derived from the Sheehan Disability Scale, which uses 3 items to assess impairments in work, family, and social functioning. ${ }^{13,29}$ The SDI is reported as an average on a 10-point Likert scale (10 indicating inability to carry out any activity).

\section{Analytic Plan}

We used an extended hot-deck multiple imputation technique that modifies the predictive mean matching method to impute item-level missing data. The strategy makes use of the well-established framework of multiple imputation, where the goal is to integrate the contribution of missing values into overall estimates of uncertainty. ${ }^{30}$ By using hot-deck imputation, imputations were restricted to values that had been observed in other subjects. Rates of item-level missing data were less than $2.5 \%$ for all variables discussed in this article. Four baseline interviews were lost at site; an approximate Bayesian boot-strap multiple imputation method was used to impute unit-level missing data for these 4 baseline surveys from screening instruments and subsequent follow-up surveys. SAS Proc MI (SAS Institute, 


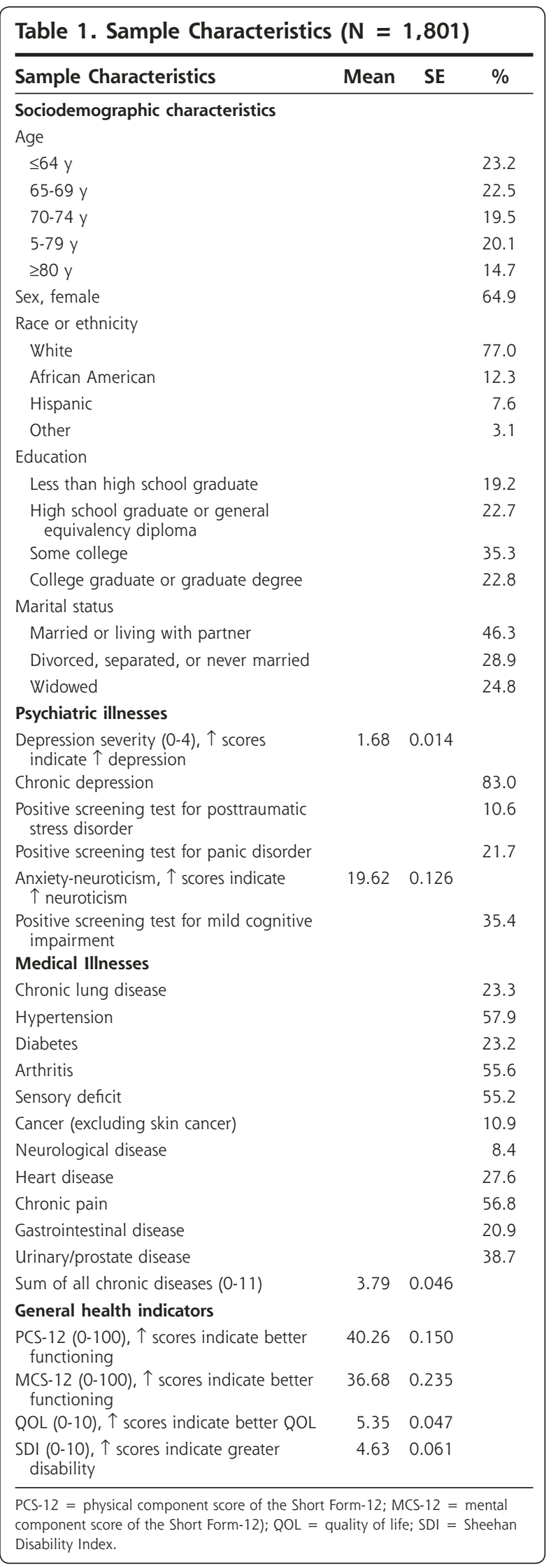

Cary, NC) was used to generate 5 imputed data sets. The MI2 SAS Macro ${ }^{31}$ was used to average regression coefficients from the 5 separate mixed-effects linear regression models. Standard errors for the regression coefficients were adjusted to reflect both within-imputation variability and between-imputation variability to achieve proper coverage. ${ }^{30}$ Simple descriptive statistics (means and standard errors for continuous variables and percentages for categorical variables) were calculated for each control variable, predictor, and outcome.

To determine which variables (sociodemographic, psychological, or medical comorbidities) were associated with the general health status measures, we conducted separate mixed-effects linear regressions using SAS PROC MIX for each outcome (MCS-12, PCS-12, SDI, and $\mathrm{QOL}$ ). In this approach, the intercept and slopes of the linear model are treated as either fixed or random effects rather than simply as a set of fixed constants, as in ordinary multiple linear or logistic regression. ${ }^{32}$ Sociodemographic factors (age, sex, ethnicity, level of education, marital status) and participating organization were entered first as fixed effects into the models as control variables. Each categorical variable having more than 2 levels was coded as a fixed effect using dummy coding. Joint tests were used to assess the significance of each categorical variable to the model. We did not include recruitment method as a predictor because it was not associated with 3 of the 4 outcomes (PCS-12, MCS-12, $\mathrm{SDI}$ ) in bivariate tests, and it did not retain significance in multivariate modeling with quality of life.

Next, the psychological variables (depression severity, chronic depression, positive screening test for panic disorder, positive screening test for posttraumatic stress disorder, neuroticism, and positive screening test for cognitive impairment) were entered as a set into the models. To determine whether other comorbid chronic medical conditions are associated with further declines in functional status, disability status, or quality of life, we then entered the set of 11 medical comorbidities. Finally, all 2-way interactions between the psychological variables and the control and medical comorbidity variables were examined and retained in the model(s), if significant. The difference in the likelihood ratio chisquare for each model tested the null hypothesis that each additional set of predictors contributed nothing beyond the set(s) of variables entered in the model(s) at earlier steps.

\section{RESULTS}

Descriptive statistics are presented in Table 1. The correlations among the 4 outcome measures (MCS-12; PCS-12, QOL, and SDI) were all significant, except for the correlation between PCS-12 and QOL (Table 2). 


\begin{tabular}{|c|c|c|c|}
\hline & MCS-12 (P Value) & QOL (P Value) & SDI ( $P$ Value) \\
\hline PCS-12 & $-0.18(<.001)$ & $0.17(.397)$ & $-0.41(<.001)$ \\
\hline MCS-12 & & $0.26(<.001)$ & $-0.24(<.001)$ \\
\hline QOL & & & $-0.30(<.001)$ \\
\hline
\end{tabular}

PCS-12 = physicial component of the Short Form-12; MCS-12 = mental component of the Short Form-12; QOL $=$ quality of life. while disability increased. Neuroticism was significantly associated with PCS-12, indicating that as neuroticism increased, physical functioning worsened. Cognitive impairment was significantly associated with physical functioning and disability. Participants with chronic lung disease, diabetes, neurological disease, heart disease, and chronic pain had significantly worse physical functioning and greater

The magnitudes of the remaining correlations were modest, ranging from 0.18 to 0.41 , indicating that the 4 outcomes measured related, albeit separate, constructs.

The models containing only the sociodemographic variables were significant $(P<.001)$ for all 4 outcome measures (MCS-12, PCS-12, QOL, and SDI). The set of psychological variables was significantly associated with all 4 outcomes (PCS-12, $P=.016$; MCS-12, $P<.001$; SDI, $P<.001$; QOL, $P<.001)$. The set of medical comorbidities contributed significant effects to the PCS-12 $(P<.001)$ and SDI $(P<.001)$ models, but not the MCS-12 $(P=.447)$ or QOL $(P=.071)$ models. Only 2 interactions significantly contributed to the models. One of the interactions suggests that African Americans with chronic depression have better mental health functioning as measured by the MCS-12 than whites with chronic depression $(P=.021)$. The second interaction suggests that as depression severity increases in patients with heart disease, their quality of life improves. This finding, however, seems somewhat counterintuitive and may be spurious in light of the marginal level of significance $(P=.041)$

Table 3 displays the final models for all 4 outcomes and indicates the significance of the difference for the likelihood ratio chi-square as each additional set of independent variables was entered into the models. Of the control variables, only organization was significantly associated with all 4 outcomes, suggesting differences in case mix at the 8 participating health care organizations. The sex of the patient was significantly associated with PCS-12 and QOL, in that men had better physical functioning than women, but worse quality of life. Level of education and ethnicity were significantly associated with PCS-12; patients with a college education had better physical functional status than those who did not graduate from high school, whereas Hispanics had better physical functional status than whites. Marital status was significantly associated with QOL, suggesting that the quality of divorced and widowed participants' life was poorer than that of married participants.

Depression severity was the only psychological variable that was significantly associated with all 4 outcomes. As depression severity increased, quality of life and physical and mental functioning declined disability. Arthritis was significantly associated with both physical and mental functioning, while hypertension and gastrointestinal disease were significantly associated with decreased physical functioning only.

Controlling for all other variables, depression severity was the only psychological or medical variable that was significantly associated with all 4 outcomes. Comparison of the standardized regression coefficients for depression severity with those of the medical illnesses indicates, however, that all 8 of the medical illnesses with significant associations (chronic lung disease, hypertension, diabetes, arthritis, neurological disease, heart disease, chronic pain, and gastrointestinal disease) contributed relatively more to physical functioning than depression severity did. Nevertheless, the standardized regression coefficients indicate that depression severity made larger independent contributions to mental health functioning, disability, and quality of life than any of the other psychological or medical variables.

\section{DISCUSSION}

We conducted a study of 1,801 elderly primary care patients with clinically severe depression to determine the relative level of association in depression severity and chronicity compared with psychiatric and medical comorbidities, on quality of life, physical functioning, mental functioning, and disability. We found that depression severity was significantly associated with all 4 indicators of general health status in this diverse sample of depressed elders. As depression severity increased, quality of life and physical and mental functioning declined, while disability increased. Furthermore, depression severity was significantly associated with all 4 indicators of health status after controlling for sociodemographic differences, other psychological conditions, and 11 medical comorbidities. Although study participants had an average of 3.8 chronic medical illnesses, depression severity made larger independent contributions to 3 of the 4 general health indicators (mental functional status, disability, and quality of life) than the medical comorbidities.

The results are somewhat surprising, given the restricted range of depression scores in this sample of 


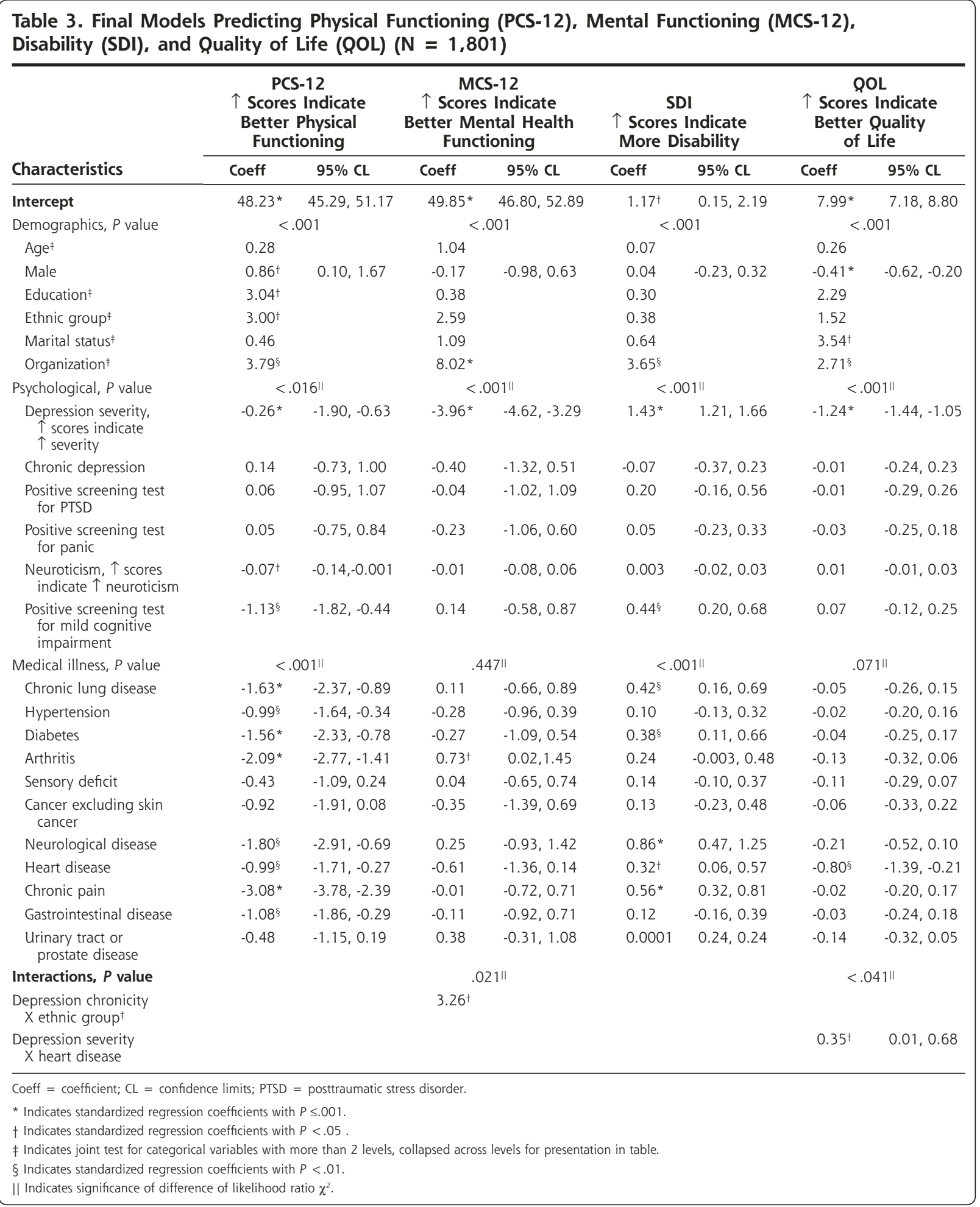

elders, all of whom met diagnostic criteria for major depressive disorder or dysthymia. It is important to note that depression severity was significantly associated with both component scores of the SF-12, considering that the scale was constructed from 2 orthogonal factors attempting to distinguish medical and mental health problems. ${ }^{14}$ The orthogonal construction artificially tends to limit the effect of a variable on both mental health and physical health components. This finding underscores the devastating impact that depres- 
sion can have on both emotional and physical functioning in older adults.

Unfortunately, depression often goes unrecognized or receives suboptimal treatment in primary care. ${ }^{33,34}$ When faced with competing demands for treating multiple chronic illnesses, physicians may give depression less priority for treatment compared with such illnesses as diabetes or arthritis. ${ }^{35-37}$ The current findings suggest, however, that depression severity is more pervasively associated with quality of life, functional status, and disability in depressed elders than most chronic medical illnesses. This association is important to recognize, because latelife depression can be successfully treated in the primary care setting with proper support. ${ }^{21,34,38,39}$ Given that chronic medical illnesses such as diabetes can often be managed only to prevent further decline, depression may well be one of our most treatable chronic illnesses among elders. Indeed, it may be that treatment for depression can lead to more dramatic improvements in functional status, disability, and quality of life than interventions for other chronic illnesses in this age-group.

This descriptive study has a number of limitations. The cross-sectional nature of the study makes it impossible to determine causality. Although the sample was recruited from 8 diverse health care organizations, the participating clinics are not representative of all primary care clinics. Although we relied upon selfreports of medical comorbidities, these were validated by medical chart review and automated data for one of the illnesses (arthritis). ${ }^{40}$ We did not, however, assess the severity of these comorbidities. The measures of health status were also derived from self-report, but these patient-centered measures have been increasingly recognized as important health outcomes.

Despite these limitations, the findings are consistent with previous research, which indicates that depression is associated with declines in a variety of general health indicators. ${ }^{8}$ Although often viewed as a sequela of medical illness, late-life depression is also related to a variety of psychosocial factors, including spousal death, role changes associated with retirement, social isolation, and diminished income. Improved recognition and treatment of depression has the potential to improve patients' lives in spite of other medical comorbidities. Future analyses from this study will determine whether multiple comorbid medical illnesses affect patient response to a collaborative treatment program for late-life depression in primary care.

\section{To read or post commentaries in response to this article, see it} online at http://www.annfammed.org/cgi/content/full/2/6/555.

Key words: Depression; primary health care; quality of life; health status

Submitted August 15, 2003; submitted, revised, January 19, 2004; accepted February 10, 2004.
Funding support: This study was supported by grants from the John A. Hartford Foundation, the California Healthcare Foundation, the Hogg Foundation, and the Robert Wood Johnson Foundation.

Acknowledgments: The IMPACT Investigators include (in alphabetical order): Patricia Arean, PhD (Co-PI); Thomas R. Belin, PhD; Noreen Bumby, DO; Christopher Callahan, MD (PI); Paul Ciechanowski, MD, MPH; Ian Cook, MD; Jeffrey Cordes, MD; Steven R. Counsell, MD; Richard Della Penna, MD (Co-PI); Jeanne Dickens, MD; Michael Getzell, MD; Howard Goldman, MD, PhD; Lydia Grypma, MD (Co-PI); Linda Harpole, MD, MPH (PI); Mark Hegel, PhD; Hugh Hendrie, MB, ChB, DSc (Co-PI); Polly Hitchcock Noël, PhD (Co-PI); Marc Hoffing, MD (PI), MPH; Enid M. Hunkeler, MA (PI); Wayne Katon, MD (PI); Kurt Kroenke MD; Stuart Levine, MD, MHA (Co-PI); Elizabeth H.B. Lin, MD, MPH (Co-PI); Tonya Marmon, MS; Eugene Oddone, MD, MHSc (Co-PI); Sabine Oishi, MSPH; R. Jerome Rauch, MD; Michael Sands, MD; Michael Schoenbaum, PhD; Rik Smith, MD; David C. Steffens, MD, MHS; Christopher A. Steinmetz, MD; Lingqi Tang, PhD; Iva Timmerman, MD; Jürgen Unützer, MD, MPH (PI); John W. Williams Jr, MD, MHS (PI); Jason Worchel, MD; Mark Zweifach, MD.

We would like to acknowledge the contributions and support of patients, primary care providers, and staff at the study coordinating center and at all participating study sites, which include: Duke University, Durham, NC; The South Texas Veterans Health Care System, The Central Texas Veterans Health Care System, and The San Antonio Preventive and Diagnostic Medicine Clinic; Indiana University School of Medicine, Indianapolis, Ind; Health and Hospital Corporation of Marion County; Group Health Cooperative of Puget Sound in cooperation with the University of Washington, Seattle, Wash; Kaiser Permanente of Northern California, Oakland and Hayward, Calif; Kaiser Permanente of Southern California, San Diego, Calif; Desert Medical Group, Palm Springs, Calif. This study is the result of work supported in part with patients, resources, and the use of facilities at the South Texas Veterans Health Care System and the Central Texas Veterans Health Care System. The views expressed in this article are those of the authors and do not necessarily represent the views of the Department of Veterans Affairs.

We would also like to acknowledge the contributions the IMPACT study advisory board (Lisa Goodale, ACSW; Richard C. Birkel, PhD; Howard Goldman, MD, PhD; Thomas Oxman, MD; Lisa Rubenstein, MD, MSPH; Cathy Sherbourne, PhD; Kenneth Wells, MD, MPH; and programming support by Tonya Marmon, MS.

\section{References}

1. Katon W. Clinical and health services relationships between major depression, depressive symptoms, and general medical illness. Biol Psychiatry. 2003;54:216-226.

2. Mancuso CA, Peterson MG, Charlson ME. Effects of depressive symptoms on health-related quality of life of asthma patients. J Gen Intern Med. 2000;15:301-310.

3. Felker B, Katon W, Hedrick SC, et al. The association between depressive symptoms and health status in patients with chronic pulmonary disease. Gen Hosp Psychiatry. 2001;23:56-61.

4. Hunkeler EM, Spector WD, Fireman B, Rice DP, Weisner C. Psychiatric symptoms, impaired function, and medical care cost in an $\mathrm{HMO}$ setting. Gen Hosp Psychiatry. 2003;25:178-184.

5. Wells KB, Stewart A, Hays RD, et al. The functioning and well-being of depressed patients: results from the Medical Outcomes Study. JAMA. 1989;262:914-919.

6. Hays RD, Wells KB, Sherbourne CD, Rogers W, Spritzer K. Functioning and well-being outcomes of patients with depression compared with chronic general medical illnesses. Arch Gen Psychiatry. 1995;52:11-19. 
7. Wells KB, Sherbourne CD. Functioning and utility for current health of patients with depression or chronic medical conditions in managed primary care practices. Arch Gen Psychiatry. 1999;56:897-904.

8. Unützer J, Patrick DL, Diehr P, Simon G, Grembowski D, Katon W. Quality adjusted life years in older adults with depressive symptoms and chronic medical disorders. Int Psychogeriatrics. 2000;12:15-33.

9. Pyne JM, Patterson TL, Kaplan RM, et al. Assessment of quality of life of patients with major depression. Psychiatric Serv. 1997;48:224-230.

10. McDowell I, Newell C. Measuring Health: A Guide to Rating Scales and Questionnaires. 2nd ed. New York, NY: Oxford University Press; 1996.

11. World Health Organization. International Classification of Impairments, Disabilities, and Handicaps. Geneva, Switzerland: World Health Organization; 1980.

12. Ware J, Sherbourne C. The MOS 36-Item Short-Form Survey (SF-36): I: conceptual framework and item selection. Med Care. 1992;30:473-483

13. Lin EH, VonKorff M, Russo J, et al. Can depression treatment in primary care reduce disability? A stepped care approach. Arch Fam Med. 2000;9:1052-1058

14. Simon GE, Revicki DA, Grothaus L, Von Korff M. SF-36 summary scores: are physical and mental health truly distinct? Med Care. 1998;36:567-572.

15. Von Korff M. Disability and psychological illness in primary care. In: Tansella M, Thornicroft G, eds. Common Mental Disorders in Primary Care: Essays in Honor of Professor Sir David Goldberg. London, England: Routledge; 1999.

16. Unützer J, Katon W, Williams JW Jr, et al. Improving primary care for depression in late life: the design of a multicenter randomized trial. Med Care. 2001; 39:785-799.

17. Spitzer RL, Williams JB, Kroenke K, et al. Utility of a new procedure for diagnosing mental disorders in primary care. The PRIME-MD 1000 Study. JAMA. 1994;272:1749-1756

18. Williams JB, Gibbon $M$, First $M B$, et al. The structured clinical interview for DSM-III-R (SCID): multisite test-retest reliability. Arch Gen Psychiatry. 1992;49:630-636.

19. First MD, Spitzer RL, Gibbon M, Williams JB. Structured Clinical Interview for DSM-IV Axis I Disorders (SCID). Washington, DC: American Psychiatric Press, Inc; 1996

20. Mayfield D, McLeod C, Hall P. The CAGE questionnaire: validation of a new alcoholism screening instrument. Am J Psychiatry. 1974; 131:1121-1123.

21. Unützer J, Katon W, Callahan CM, et al. Collaborative care management of late life depression in the primary care setting: a randomized controlled trial. JAMA. 2002;288:2836-2845.

22. Derogatis LR, Lipman RS, Covi L. SCL-90: an outpatient psychiatric rating scale. Psychopharmacol Bull. 1973;9:13-28.

23. Rush J et al. The inventory for depressive symptomatology (IDS): preliminary findings. Psychiatry Res. 1985;18:65-87.
24. Costa PT, McCrae RR. The NEO Personality Inventory Manual. Odessa Fla: Psychological Assessment Resources; 1985

25. Hegel MT, Unutzer J, Tang L, et al. Impact of co-morbid panic disorder and PTSD on outcomes of collaborative care for late-life depression in primary care. Am J Geriatr Psychiatry. In press.

26. Callahan CM, Unverzagt FW, Hui SL, et al. Six-item screener to identify cognitive impairment among potential subjects for clinical research. Med Care. 2002:40:771-781.

27. Ware JE, Kosinski M, Keller S. A 12-item short form health survey: construction of scales and preliminary tests of reliability and validity. Med Care. 1996;34:220-233.

28. Froberg DG, Kane RL. Methodology for measuring health-state preferences - IV: progress and a research agenda. J Clin Epidemiol. 1989;42:675-685.

29. Sheehan DV, Harnett-Sheehan K, Raj BA. The measurement of disability. Int Clin Psychophamacol. 1996;11(suppl 3):89-95.

30. Rubin DB. Multiple Imputation for Non-response in Surveys. New York, NY: J Wiley \& Sons; 1987.

31. M12.mac [computer program]. Macros for multiple imputation. Santa Monica, Calif: RAND Corporation; 2000

32. Littell RC, Milliken GA, Stroup WW, Woltinger RD. SAS System for Mixed Models. Cary, NC: SAS Institute Inc; 1996.

33. Unützer J, Katon W, Sullivan M. Treating depressed older adults in primary care: narrowing the gap between efficacy and effectiveness. Milbank Q. 1999;77:225-256.

34. Hunkeler EM, Meresman JF, Hargreaves WA, et al. Efficacy of nurse telehealth care and peer support in augmenting treatment of depression in primary care. Arch Fam Med. 2000;9:700-708.

35. Williams JW Jr, Rost K, Dietrich AJ, Ciotti MC, Zyzanski SJ, Cornell J. Primary care physicians' approach to depressive disorders. Effects of physician specialty and practice structure. Arch Fam Med. 1999;8:58-67.

36. Rost K, Humphrey J, Kelleher K. Physician management preferences and barriers to care for rural patients with depression. Arch Fam Med. 1994:3:409-414

37. Klinkman, MS. Competing demands in psychosocial care. A model for the identification and treatment of depressive disorders in primary care. Gen Hosp Psychiatry. 1997;19:98-111.

38. Katon W, Von Korff M, Lin E, et al. Stepped collaborative care for primary care patients with persistent symptoms of depression: a randomized trial. Arch Gen Psychiatry. 1999;56:1109-1115.

39. Katon W, Von Korff M, Lin E, et al. Collaborative management to achieve treatment guidelines: impact on depression in primary care. JAMA. 1995;273:1026-1031.

40. Lin EH, Katon W, Von Korff $M$, et al, for the IMPACT investigators. Effect of improving depression care on pain and functional outcomes among older adults with arthritis: a randomized controlled trial. JAMA. 2003;290:2428-2429. 